\title{
Variabilidad y cambios climáticos observados y esperados en el Altiplano del norte de Chile ${ }^{1}$
}

\author{
Pablo Sarricolea Espinoza² y Hugo Romero Aravena ${ }^{3}$
}

\begin{abstract}
RESUMEN
Se estudian a distintas escalas temporales y espaciales, las temperaturas, irregularidad de las precipitaciones y sequías en el Altiplano del norte de Chile mediante datos provenientes de estaciones meteorológicas y coberturas climáticas de alta resolución espacial, en relación a las condiciones actuales y futuras derivadas del Quinto Informe del IPCC, con el propósito de realizar un análisis y evaluación de la variabilidad y cambios climáticos que afectan a esta región del país. Se confirma el aumento de la temperatura, y se consigue precisar a escala regional la disminución esperada para las precipitaciones, así como el aumento de su variabilidad. Se han identificado distintos territorios de respuesta al cambio climático en el Altiplano, considerando la variabilidad espacial y temporal de las temperaturas y las precipitaciones medias y extremas (Iluvia y sequías). Los resultados demuestran la existencia de un coeficiente de variación alto de las precipitaciones anuales y de respuestas contradictorias usando el índice de concentración de la precipitación diaria.
\end{abstract}

Palabras clave: Altiplano andino, cambio climático, índice de concentración, precipitación diaria, variabilidad climática

\begin{abstract}
This study analyses temperature, rainfall irregularity and droughts in the Altiplano of Northern Chile at different temporal and spatial scales using data from meteorological stations and high spatial resolution climatic coverage, in respect to current and future scenarios included in the Fifth IPCC report. The aim of this work is to analyze and evaluate climate variability and climate change affecting this region. Our work confirms an increase in temperature, and offers regional scale precision as to the predicted reduction in rainfall, as well as its predicted increase in variability. We identified territories with distinct responses to climate change in the Altiplano, considering spatial and temporal variability in temperature, rainfall and extreme events (storms and droughts). Our results indicate a high coefficient of variation in annual rainfall and an opposite response when the concentration index for daily precipitation is considered.
\end{abstract}

Key words: Andean highlands, climate change, climate variability, daily precipitation, concentration index.

\footnotetext{
1 Investigación financiada por los proyectos FONDECYT Regular No 1120204 y No 1150701. Artículo recibido el 5 de marzo de 2014 , aceptado el 10 de octubre de 2014 y corregido el 12 de mayo de 2015.
}

\footnotetext{
2 Departamento de Geografía, Universidad de Chile (Chile). E-mail: psarricolea@uchilefau.cl

3 Departamento de Geografía, Universidad de Chile (Chile). E-mail: hromero@uchilefau.cl
} 
El extremo nororiental de Chile continental forma parte del Altiplano sudamericano (Fuenzalida y Rutlant, 1987), una meseta de los Andes centrales que supera los 3.000 m.s.n.m., y que es compartida entre Argentina, Bolivia, Chile y Perú (Figura $\left.N^{\circ} 1 \mathrm{~A}\right)$. Se localiza entre los $15^{\circ} \mathrm{S}$ y los $22^{\circ} \mathrm{S}$ (Minvielle \& Garreaud, 2011), correspondiendo administrativamente a las regiones XV de Arica y Parinacota, I de Tarapacá y II de Antofagasta, aunque la Dirección General de Aguas (DGA) lo extiende de modo discontinuo hasta la III Región de Atacama $\left(27^{\circ} \mathrm{S}\right)$ (Figura $\mathrm{N}^{\circ} 1 \mathrm{~B}$ ). El Altiplano posee una alta radiación solar, facilitada por su Ilana topografía, posición latitudinal y la menor capacidad de absorción que confiere el escaso espesor atmosférico superior (Aceituno, 1996). Además, se sitúa entre el desierto más árido del mundo (en la costa del Pacífico) y la cuenca amazónica por el oriente, la que se constituye como una región continental húmeda. Posee un clima semiárido, con precipitaciones que decrecen de norte a sur (600-200 mm anuales), concentradas entre diciembre y marzo (60-90\% del total anual), y diariamente durante la ocurrencia de tormentas convectivas que se activan localmente por bajas térmicas, que se desarrollan especialmente en las tardes (Garreaud et al., 2003). Para efectos de este estudio se analizan las regiones XV de Arica y Parinacota y I de Tarapacá (Figura $N^{\circ} 1 \mathrm{C}$ ), las cuales poseen entre sus actividades económicas de mayor importancia a la minería.

Las precipitaciones del Altiplano tienen una alta irregularidad, tanto a escalas intraestacional e interanual (Romero et al., 2013), caracterizadas por la alternancia de episodios húmedos y secos que en el verano poseen una frecuencia de 5 a 15 días (Falvey \& Garreaud, 2005). Varios estudios (Garreaud \& Aceituno, 2001; Romero et al., 2013) señalan que una fracción significativa de la variabilidad interanual de las precipitaciones sobre el Altiplano está relacionada con El Niño Oscilación del Sur (ENSO), condicionando la ocurrencia de episodios húmedos con la fase fría de este evento (años La Niña) y de episodios secos que se registran durante la fase cálida (años El Niño). De modo intraestacional, las precipitaciones del Altiplano en verano son dominadas por el exceso de calor latente en la atmósfera, que duplica al calor sensible aportado por el suelo, generando inestabili- dad de la columna de aire (Fuenzalida \& Rutlant, 1987); esto es causa de la configuración y variabilidad sinóptica de la Zona de Convergencia Intertropical (ZCIT), la activación del sistema del Monzón Sudamericano y la conformación cuasi-estacional del anticiclón cálido en altura (200 hPa) denominado "Alta de Bolivia", además de los fenómenos locales de calentamiento diurno de la superficie terrestre. No obstante, la influencia de los sistemas sinópticos que afectan el tiempo atmosférico del Altiplano no actúan de modo homogéneo, variando su influencia según la latitud, altitud, cuenca hidrográfica y orientación de las laderas (Romero et al., 2013).

Por otra parte, las temperaturas medias en el Altiplano andino son relativamente bajas, conformando un factor bioclimático limitante para el desarrollo de la vegetación (Aceituno, 1993). Basta recordar que en atmósfera libre la temperatura desciende a una tasa cercana a $6,5^{\circ} \mathrm{C} / \mathrm{km}$, y se han registrado gradientes de la temperatura en superficie de unos $5,5^{\circ} \mathrm{C} /$ km en el Altiplano de Perú (Frère et al., 1975, en: Aceituno, 1993).

Los cortos y escasos registros de que se dispone en las series climáticas de estaciones meteorológicas del Altiplano, no permiten establecer tendencias claras durante el siglo $X X$, referidas a aumentos o disminuciones interanuales significativos de las temperaturas y las precipitaciones; por el contrario, más bien reflejan una alta irregularidad con alzas y disminuciones asociadas en parte al ENSO. No obstante, los modelos climáticos globales (GCM) y los regionales (RCM) construidos bajo los escenarios de emisiones de gases de efecto invernadero y variabilidad natural, proporcionados por los informes de evaluación (Assessment Report, en siglas AR) Tercero y Cuarto del Panel Intergubernamental de Cambio Climático (Intergovernmental Panel on Climate Change, en siglas IPCC en inglés) señalan para el Altiplano, tendencias de aumentos significativos de las temperaturas, y escasos niveles de confianza sobre las tendencias de las precipitaciones a finales del siglo XXI (Fuenzalida et al., 2007), pese a que en Minvielle y Garreaud (2011) se menciona un debilitamiento de los flujos desde la cuenca amazónica, y por lo tanto, una disminución de las precipitaciones, lo cual es consistente con los resultados publicados por 

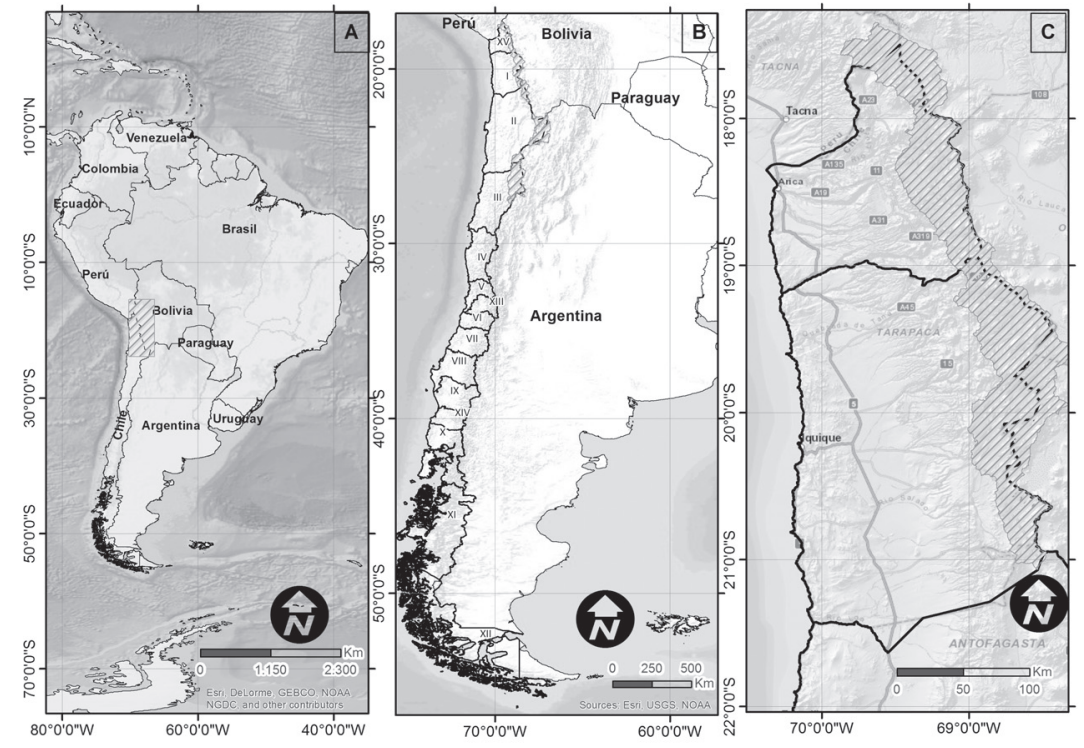

Fuente: Elaboración propia en base a cuencas altiplánicas de la Dirección General de Aguas.

el Quinto Informe de Evaluación (AR5) del IPCC (2013: 1264).

Es por ello, que la importancia de analizar las precipitaciones en su doble dimensión (presencia y ausencia) y el comportamiento de las temperaturas, obedece a que el Altiplano no es exclusivamente afectado por el Cambio Climático Global (CCG), sino que también por la cada vez mayor presión de las actividades mineras, las cuales demandan recursos hídricos, que provienen de cursos de aguas superficiales y acuíferos subterráneos. Otro aspecto a resaltar es que los modelos climáticos globales de circulación atmosférica son cada vez más robustos, y el AR5 (IPCC, 2013), destaca para el Hemisferio Sur, que se ha conseguido reducir de modo muy significativo la incertidumbre, fundamentalmente en detectar y pronosticar tendencias de las precipitaciones.

Las proyecciones climáticas obtenidas de los modelos climáticos globales se basan en escenarios representativos de emisiones (en inglés RCP o representative concentration pathways), definidos según grados de forzamientos radiativos y concentraciones atmosféricas de gases de efecto invernadero (GEI); es decir, corresponden a una prognosis de gran escala, largo plazo y ámbito planetario, por lo que sus resultados no pueden ser usados directamente para estudios de impactos regionales, sino que deben ser acompañados de un escalamiento hacia abajo o downscaling (Wilby \& Wigley, 1997). Un downscaling corresponde geográficamente a una interpolación de moderada o alta resolución espacial $(25 \times 25 \mathrm{~km}$ o $1 \times 1 \mathrm{~km})$ de los escenarios considerados por los modelos climáticos globales (de los cuales depende y se anidan), favorecidos por múltiples fuentes de información: una mayor cobertura y densidad de estaciones meteorológicas, radiosondeos, reanálisis y otras informaciones del área geográfica de interés (modelos digitales de elevaciones, albedos de las coberturas y usos de suelo, tipo y estado de la vegetación, etc.). Los downscaling pueden ser estadísticos o dinámicos, diferenciados por la mayor complejidad matemática y física que poseen entre ellos. 
Desde el punto de vista climático, es sabido que un monto o promedio mensual de precipitación suministra información valiosa sobre la pluviometría de un territorio, pero no dice nada acerca de la frecuencia o número de días de lluvia, ni tampoco del desglose de las cantidades registradas en los días lluviosos (Sarricolea y Martín-Vide, 2012). Cabe destacar que esta situación es mucho más simple en el caso de las temperaturas, las cuales poseen marcados ritmos diarios, mensuales e incluso interanuales. La variabilidad temporal de las precipitaciones ha sido usualmente calculada y resumida a nivel mensual, estacional y anual en las series climáticas mediante distintos estadígrafos. Por ejemplo, la varianza, el coeficiente de variación (CV), la desviación estándar y, más recientemente, por el índice de irregularidad temporal (S1, propuesto por Martín-Vide et al., 2001). Este último índice considera en contraste con los antes mencionados, el orden de los valores en la serie y las diferencias expresadas de modo absoluto. Sin embargo, cabe hacer notar que todos estos parámetros estadísticos que expresan la variabilidad de las precipitaciones se aplican casi siempre sobre montos mensuales o anuales y raramente a nivel diario.

Martín-Vide $(1984,2004)$ formuló el Índice de Concentración ( $\mathrm{Cl}$ o Concentration Index, en adelante) para estudiar las precipitaciones diarias. El $\mathrm{Cl}$ es un indicador sintético que permite calcular el grado de concentración de las precipitaciones. Además, es un estimador del grado de agresividad o torrencialidad pluviométrica.

$\mathrm{El} \mathrm{Cl}$ indica en Chile que las estaciones de Arica, Antofagasta, Iquique y Puerto Montt presentan valores menores o iguales que 0,57 (Sarricolea y Martín-Vide, 2012). El Cl alcanza sus máximos valores en La Serena $(0,76)$, seguido de Copiapó $(0,68)$, Valparaíso $(0,64)$ y Santiago $(0,63)$. En resumen, el $\mathrm{Cl}$ máximo se consigue en Chile central, justamente donde el clima es mediterráneo. A partir de Chile central, el $\mathrm{Cl}$ decrece hacia las áreas desérticas del norte y templadas lluviosas del sur. Visto con más detalle, la situación antes descrita se reproduce en Chile central (Sarricolea et al., 2013), mostrando claras dependencias a componentes zonales (latitudinales) y meridianas (orográficas). En el Altiplano andino como una región semiárida, se pretende conocer qué ocurre, y si se alcanzan valores de $\mathrm{Cl}$ similares a Chile central, pues sus montos pluviométricos anuales son muy similares, no así el origen de las precipitaciones (frontal versus convectiva) ni estacionalidad (invierno versus verano). Otros indicadores para estudiar la precipitación diaria serán las rachas de sequía (longitud de días de ausencia de lluvia) y los períodos de retorno, los cuales entregan valiosa información de fenómenos extremos que pueden desencadenar riesgos socionaturales. Los extremos en un escenario de Cambio Climático son fundamentales, pues Karl et al. (2008) señalan una disminución de los días de lluvia poco cuantiosa (es decir, más días secos) y un aumento de los días de precipitación máxima extrema.

Por ello, las preguntas que subyacen esta investigación son: ¿La variabilidad actual de las precipitaciones muestran alguna tendencia? ¿Cuáles son (de existir) las tendencias y patrones espaciales de la precipitación y la temperatura hacia finales del siglo XXI en el Altiplano? ¿Es posible aportar nuevos conocimientos sobre la disponibilidad de agua en el Altiplano?; y finalmente, ¿qué se verá más agravado, la precipitación máxima extrema o la sequía?

\section{Materiales y métodos}

Para responder a dichas preguntas se examinan la precipitación diaria de 11 estaciones meteorológicas de la Dirección General de Aguas (1984-2010), cubiertas climáticas mensuales de precipitación y temperatura del período 1950-2000 (Hijmans et al., 2005; Pliscoff et al., 2014) y del AR5 en su escenario más adverso (RCP8.5) para el 2070 (20612080), y a una resolución downscaling de $1 \times 1 \mathrm{~km}$.

\section{Análisis de las precipitaciones y sequía}

Para la evaluación del clima actual en el Altiplano se utilizaron fuentes instrumentales de la precipitación para los últimos años (1984-2010), correspondientes a las estaciones meteorológicas de la Dirección General de Aguas, las cuales se presentan en el Cuadro № 1 . Con ellas se construyeron ín- 
dices, tales como el de longitud diaria de las rachas de sequía, irregularidad temporal (S1), coeficiente de variación (CV) y el índice de concentración $(\mathrm{Cl})$. Además, se calcularon los períodos de retorno de precipitación máxima de 1, 2 y 3 días.

Para determinar longitudes de las rachas de sequía, se consideró el número promedio de días secos para cada año en el período estival (diciembre a marzo). Para estudiar las precipitaciones diarias y la serie en su totalidad. Se prefiere este índice absoluto (MartínVide \& Gómez, 1999) a otros de persistencia de la sequía como el de Besson o las Cadenas de Markov, por su fácil proyección futura en escenarios de cambio climático.

Se calcularon para las precipitaciones de cada una de las 11 estaciones meteorológicas

\section{Cuadro $\mathrm{N}^{\circ} 1$}

Estaciones meteorológicas utilizadas, sus códigos DGA, coordenadas geográficas, altitud y localización administrativa

\begin{tabular}{|c|c|c|c|c|c|c|c|}
\hline $\begin{array}{l}\text { Código } \\
\text { DGA }\end{array}$ & $\begin{array}{l}\text { Nombre } \\
\text { estación }\end{array}$ & Latitud (S) & Longitud (W) & $\begin{array}{c}\text { Altitud } \\
\text { (m.s.n.m.) }\end{array}$ & Región & Provincia & Comuna \\
\hline $01000005-K$ & Visviri & $17^{\circ} 35^{\prime} 54^{\prime \prime}$ & $69^{\circ} 29^{\prime} 5,5^{\prime \prime}$ & 4.080 & \multirow{8}{*}{$\begin{array}{c}\text { Arica y } \\
\text { Parinacota }\end{array}$} & \multirow{8}{*}{ Parinacota } & $\begin{array}{c}\text { General } \\
\text { Lagos }\end{array}$ \\
\hline 01001005-5 & Caquena & $18^{\circ} 3^{\prime} 26,9^{\prime \prime}$ & $69^{\circ} 12^{\prime} 12,9^{\prime \prime}$ & 4.400 & & & \multirow{7}{*}{ Putre } \\
\hline 01010007-0 & $\begin{array}{l}\text { Chungará } \\
\text { Retén }\end{array}$ & $18^{\circ} 17^{\prime} 16^{\prime \prime}$ & $69^{\circ} 8^{\prime} 17,6^{\prime \prime}$ & 4.570 & & & \\
\hline 01010010-0 & $\begin{array}{l}\text { Chungará } \\
\text { Ajata }\end{array}$ & $18^{\circ} 14^{\prime} 19,2^{\prime \prime}$ & $69^{\circ} 11^{\prime} 6,8^{\prime \prime}$ & 4.585 & & & \\
\hline 01020014-8 & Cotacotani & $18^{\circ} 11^{\prime} 12,6^{\prime \prime}$ & $69^{\circ} 14^{\prime} 7,1^{\prime \prime}$ & 4.550 & & & \\
\hline 01020016-4 & $\begin{array}{l}\text { Chucuyo } \\
\text { Retén }\end{array}$ & $18^{\circ} 13^{\prime} 2,5^{\prime \prime}$ & $69^{\circ} 19^{\prime} 24,4^{\prime \prime}$ & 4.400 & & & \\
\hline 01020017-2 & $\begin{array}{l}\text { Parinacota } \\
(\text { ex Endesa) }\end{array}$ & $18^{\circ} 12^{\prime} 15^{\prime \prime}$ & $69^{\circ} 16^{\prime} 6,6^{\prime \prime}$ & 4.420 & & & \\
\hline 01021007-0 & Guallatire & $18^{\circ} 29^{\prime} 49,5^{\prime \prime}$ & $69^{\circ} 9^{\prime} 15,2^{\prime \prime}$ & 4.240 & & & \\
\hline 01041003-7 & $\begin{array}{l}\text { Colchane } \\
\text { (Tte. Isluga) }\end{array}$ & $19^{\circ} 16^{\prime} 19,4^{\prime \prime}$ & $68^{\circ} 38^{\prime} 28,3^{\prime \prime}$ & 3.700 & \multirow{3}{*}{ Tarapacá } & \multirow{3}{*}{ Tamarugal } & Colchane \\
\hline 01042001-6 & Cancosa & $19^{\circ} 51^{\prime} 30,6^{\prime \prime}$ & $68^{\circ} 36^{\prime} 13^{\prime \prime}$ & 3.930 & & & \multirow{2}{*}{ Pica } \\
\hline 01050007-9 & Coyacagua & $20^{\circ} 2^{\prime} 15,9^{\prime \prime}$ & $68^{\circ} 49^{\prime} 48,7^{\prime \prime}$ & 3.950 & & & \\
\hline
\end{tabular}

Fuente: Elaboración propia en base a la Dirección General de Aguas.

los promedios anuales, la Desviación Típica, el Coeficiente de Variación (CV) y el Índice de Irregularidad Temporal $\left(\mathrm{S}_{1}\right)$. El $\mathrm{S}_{1}$ considera la secuencia de los datos en serie, tal como advierte Martín-Vide (2003), y se construye a partir de la sumatoria resultante de los cocientes de las diferencias absolutas de las precipitaciones de años sucesivos (transformados a logaritmos neperianos), dividido el valor por la longitud de la serie menos una unidad, tal como se observa en siguiente expresión:

$$
S_{1}=\left[\sum\left|\ln \left(P_{i+1} / P_{i}\right)\right| /(n-1)\right]
$$

Para la obtención del índice de concentración, se ha trabajado con la resolución diaria de la serie. Las precipitaciones se han agru- 
pado en clases de $1 \mathrm{~mm}$, y se ha procedido a contar el número de días de lluvia de cada clase y estación. El $\mathrm{Cl}$ se expresa en valores entre 0 y 1 , y corresponde a la proporción del área entre la curva del ajuste exponencial y la diagonal del cuadrado de lado 100 o 10.000 unidades de área. Por lo tanto, el $\mathrm{Cl}$ se calcula como la proporción de área bajo la diagonal $\left(S^{\prime} / 5.000\right)$, y para obtenerla se debe conocer el área inferior a la curva exponencial $\left(A^{\prime}\right)$. Dicho ajuste exponencial expresa el porcentaje de días de lluvia y sus totales, ambos acumulados, lo cual se puede revisar en Martín-Vide (2004) y en Sarricolea et al. (2013).

Para el cálculo de los períodos de retorno se usó la función de Gumbel, la cual cuenta con dos parámetros ( forma y escala), calculados a partir de la media y desviación típica), tal como se aprecia en la siguiente expresión:

$$
f(x)=\exp (-\exp (-((x-u) / \alpha)))
$$

Ambas funciones se pueden llevar a períodos de retorno, pues se corresponden con la función inversa de la probabilidad (1/). En el caso de Gumbel por ejemplo, queda de la siguiente manera:

$$
T=\frac{1}{1-f\left(x_{T}\right)}
$$

Los eventos máximos de variables discretas (como es el caso de las precipitaciones) pueden asumir diversas distribuciones que tienen modelos exponenciales como común denominador, siendo uno de los más utilizados el que posee una distribución de Gumbel (Clarke, 2002). Para establecer si la pluviometría de máximas sigue dicha distribución se realizó el test de hipótesis del Chi Cuadrado.

Además, entre las variables analizadas se realizaron regresiones lineales con el propósito de estimar los posibles cambios hacia el futuro.

\section{Análisis de la variabilidad y cambio climático}

Una de las primeras fuentes consideradas para el análisis fue el modelo climático regio- nal elaborado con Providing Regional Climates for Impact Studies o PRECIS (Fuenzalida et al., 2007), el que posee una resolución espacial de salida de $25 \times 25 \mathrm{~km}$. Este modelo fue anidado bajo las condiciones establecidas por el modelo climático global "HadAM3P" (del AR3 del IPCC), y corresponde a uno de los escenarios moderados. Entendiendo los grandes problemas que dicho modelo ha demostrado en el Altiplano, su escasa resoIución espacial, y teniendo en cuenta que en 2013 se dispone del Quinto Informe del IPCC (AR5), se procedió a utilizar uno de tales modelos en versión downscaling, y para una resolución espacial de $1 \times 1 \mathrm{~km}$. El modelo utilizado está inserto en la quinta fase de modelos acoplados del IPCC (Coupled Model Intercomparison Project Phase 5, CMIP5), y corresponde al Australian Community Climate and Earth System Simulator (ACCESS 1.0) desarrollado conjuntamente por la Organización para la Investigación Científica e Industrial de la Mancomunidad Australiana (Commonwealth Scientific and Industrial Research Organisation CSIRO) y la Oficina Australiana de Meteorología (Bureau of Meteorology BOM).

El modelo acoplado ACCESS 1.0 es amplia y detalladamente descrito en Bi et al. (2013), y en numerosos artículos de la edición especial 2013 del Australian Meteorological and Oceanographic Journal. ACCESS 1.0 se compone de modelos de la atmósfera, el manto de hielo y nival, de coberturas y usos de suelo, modelos de circulación oceánica y del sistema acoplado océano-hielo, los que han sido unificados en su mayoría por la Met-Office (UK Meteorological Office). ACCESS 1.0 fue desarrollado para dos escenarios futuros, el RCP4.5 y RCP8.5, los cuales representan forzamientos radiativos de tipo moderado-bajo y alto $\left(4,5 \mathrm{~W} / \mathrm{m}^{2}\right.$ y $\left.8,5 \mathrm{~W} / \mathrm{m}^{2}\right)$ y concentraciones de $\mathrm{CO}_{2}$ para el año 2100 equivalentes a 650 y 1370 ppm respectivamente. Para esta investigación se trabajó con el forzamiento radiativo alto, pues es el peor escenario posible, y el mejor para ilustrar los posibles cambios climáticos.

Las variables utilizadas del ACCESS 1.0 en el escenario RPC8.5 fueron la temperatura media anual, la precipitación anual y el coeficiente de variación de la precipitación, denominadas superficies bioclimáticas BIO1, 
BIO12 y BIO15. Ellas, representan la climatología mundial esperada para el período 2061-2080, y fueron contrastadas de modo comparativo con las mismas superficies climáticas BIO1, BIO12 y BIO15 creadas para Sudamérica por Pliscoff y Luebert (2013), para el período 1950-2000.

Con el propósito de avaluar la variabilidad y cambios climáticos se utilizó el método delta o de diferencias. A partir de esto, se obtuvieron anomalías absolutas de temperatura (BIO1 $1_{\text {futuro }}$-BIO1 $\left.1_{\text {actual }}\right)$ y anomalías porcentuales de las precipitaciones ((BIO12 futuro- $^{-}$ BIO12 actual)/ BIO12 futuro $_{\text {}}$. En el caso de la variabilidad (BIO15) no fue necesario el uso del método delta, pues es más gráfico presentar sus resultados de modo comparado.

Para clasificar los cambios en las temperaturas y en los montos de precipitación se consideró el estudio de la Universidad de Chile "ACT-19, el clima de Chile está cambiando4", en el cual, los cambios menores a $2^{\circ} \mathrm{C}$ son considerados débiles, entre 2 y $4^{\circ} \mathrm{C}$ como moderados, entre $4 \mathrm{y}^{\circ} \mathrm{C}$ son calificados como fuertes y sobre $6^{\circ} \mathrm{C}$ como muy fuertes. En el caso de las precipitaciones, cambios sobre y bajo $15 \%$ son considerados fuertes.

\section{Análisis de las precipitaciones y la sequía}

Como se aprecia en el Cuadro $\mathrm{N}^{\circ} 2$, las precipitaciones disminuyen (en general) desde el norte al sur, pero con algunos matices, pues las precipitaciones medias máximas se alcanzan en la estación de Cotacotani, y las medias mínimas en Colchane. Estas medias encubren la alta variabilidad que las precipitaciones alcanzan en el Altiplano (Romero et al., 2013), lo cual tampoco es resuelto por la interpretación de la desviación estándar, pus no aporta de modo significativo órdenes de magnitud de la variabilidad. Sin embargo, el Coeficiente de Variación resulta ser un buen indicador de la variabilidad de las series pluviométricas y la comparación entre ellas. Además muestra un Coeficiente de Correlación de tipo negativo para las precipitaciones anuales medias con un $r=-0,81$, lo que apli-

Cuadro $\mathrm{N}^{\circ} 2$

Síntesis de resultados de los datos analizados de precipitación media anual (P), Desviación Estándar, Coeficiente de Variación (CV), Índice de Concentración (CI), Longitud de la Sequía (L) e Irregularidad Temporal (S1) entre 1984-2010

\begin{tabular}{|l|r|r|r|r|r|r|}
\hline Nombre estación & $\mathrm{P}(\mathrm{mm})$ & Desviación estándar & $\mathrm{CV}(\%)$ & $\mathrm{Cl}$ & $\mathrm{L}($ días $)$ & $\mathrm{S} 1$ \\
\hline Visviri & 263,10 & 151,29 & 58 & 0,45 & 40,04 & 0,47 \\
Caquena & 381,04 & 194,79 & 51 & 0,44 & 13,74 & 0,50 \\
Chungará Retén & 272,95 & 118,32 & 43 & 0,43 & $\mathrm{~s} / \mathrm{i}$ & 0,61 \\
Chungará Ajata & 380,10 & 134,68 & 35 & 0,45 & 14,19 & 0,35 \\
Cotacotani & 427,90 & 152,43 & 36 & 0,43 & 15,04 & 0,36 \\
Chucuyo Retén & 345,56 & 147,91 & 43 & 0,44 & 32,07 & 0,62 \\
Parinacota (ex Endesa) & 301,52 & 142,52 & 47 & 0,50 & 17,89 & 0,39 \\
Guallatire & 248,76 & 126,23 & 51 & 0,44 & 23,63 & 0,47 \\
Colchane (Tte. Isluga) & 124,01 & 70,89 & 57 & 0,47 & 44,56 & 0,69 \\
Cancosa & 153,09 & 117,79 & 77 & 0,46 & 93,00 & 0,81 \\
Coyacagua & 130,35 & 82,83 & 64 & 0,50 & 59,26 & 0,61 \\
\hline
\end{tabular}

Fuente: Elaboración propia.

\footnotetext{
4 Disponible en http://www.dgf.uchile.cl/ACT19/html/ avances.html
} 
cando el T test permite aceptar la hipótesis nula (no existen diferencias significativas a un alpha de 0,05), y con ello predecir cambios mediante análisis de regresión. Por otro lado, la distribución espacial del Coeficiente de Variación indica que Cancosa posee el mayor valor (77\%), seguida de Coyacagua (64\%) y Visviri (58\%), mientras que los valores más bajos de CV se localizan en Chungará y Cotacotani (35\% y 36\% respectivamente).

Por otra parte, el Índice de Concentración (CI), que al igual que el Coeficiente de Variación da cuenta de la variabilidad de las precipitaciones, arroja valores bajos en toda la región, incluso si se le compara con otras zonas planetarias o del territorio nacional. Sus valores extremos están entre 0,43 y 0,50 (ver Cuadro $N^{\circ} 2$ y Figura $N^{\circ} 2$ ). Espacialmente permite separar a una latitud de $19^{\circ} 30^{\prime}$ S, una región de muy baja irregularidad al norte (valores menores a 0,46$)$ y una de baja irre- gularidad pluviométrica al sur. Es muy peculiar, pero se puede interpretar del siguiente modo: "en el Altiplano del Norte de Chile las precipitaciones se registran casi en forma exclusiva en la estación estival, y la distribución de ellas, expresada en clases de $1 \mathrm{~mm}$ están bien equidistribuidas, no como en Chile central". Sin duda, el origen convectivo de las precipitaciones, y la activación de procesos de variabilidad de baja frecuencia favorecen que precipite de modo regular en el verano, manteniendo ciclos de 5-15 días (Falvey \& Garreaud, 2005) y siguiendo secuencias alternadas de años lluviosos y secos en todo el Altiplano, pudiendo alcanzar hasta 5 años consecutivos de sequía meteorológica, es decir bajo la normal y períodos de tres años seguidos de lluvia (Figura $N^{\circ} 3$ ). Si dichos patrones representan la variabilidad actual es posible esperar que ellos sean más extensos en el futuro.

Figura $\mathrm{N}^{\circ} 2$

Índice de concentración de las precipitaciones en el Altiplano del norte de Chile (1984-2010)

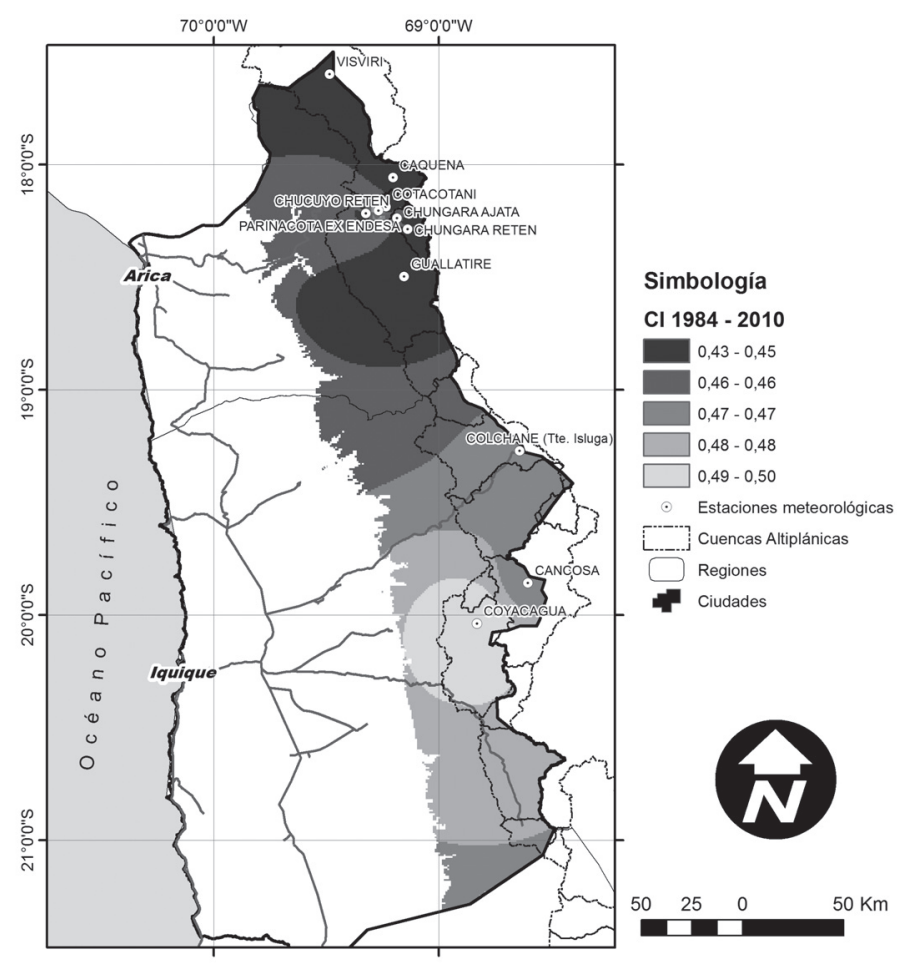

Fuente: Elaboración propia. 
Figura $\mathrm{N}^{\circ} 3$

Clasificación de años Iluviosos y secos según puntajes Z en el Altiplano del norte de Chile (1984-2010)

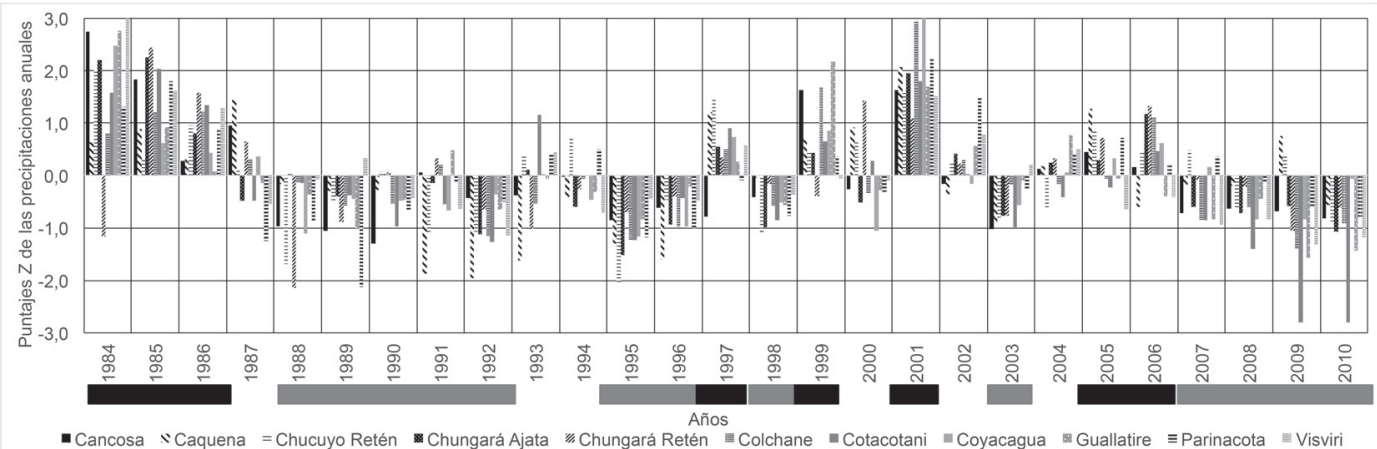

Fuente: Elaboración propia. La barra negra muestra años lluviosos y la gris años secos.

Al observar en el Cuadro $N^{\circ} 2$ la longitud media de los períodos sin precipitación estival $(<0,1 \mathrm{~mm})$ es posible establecer que son más extensos hacia el sur, registrándose hasta 44 días sin Iluvia en el verano. Al correlacionar la sequía con las otras variables del Cuadro $\mathrm{N}^{\circ} 1$ se vuelve a encontrar un Coeficiente de Correlación alto con el CV $(r=0,90)$, y positivo, es decir, que a mayor CV mayor longitud de la racha de sequía, lo que queda respaldado por los test de significancia estadísticas. De este modo, si en el futuro, producto del cambio y variabilidad climática, aumenta el Coeficiente de Variación, las rachas diarias de sequías estivales se verán incrementadas.

En relación a la irregularidad consecutiva o temporal de las precipitaciones $\left(\mathrm{S}_{1}\right)$ se advierte una diferencia respecto a las otras variables, pues ella se correlaciona mejor con la longitud de la sequía (en vez del Coeficiente de Variación) registrando un $r=0,87$. De este modo, el $\mathrm{S}_{1}$ aumenta meridionalmente en el Altiplano del norte de Chile, al igual que la sequía.

Resulta interesante que en general, la mayoría de las variables analizadas de la serie pluviométrica diaria entre 1984-2010 señalen una alta variabilidad de las precipitaciones, con la excepción única del índice de concentración $(\mathrm{Cl})$. Pese a ello, la consistencia climática entre las variables es alta, geográ- ficamente consistente y congruente con la literatura referida al Altiplano.

\section{Resultados del análisis de períodos de retorno}

Los procesos de remoción en masa en el Altiplano del norte de Chile están modulados por precipitaciones intensas de uno o varios días, y que por lo general son de tipo convectivo. Dichas precipitaciones se pueden segmentar en días e incluso horas de ocurrencia. No obstante, ha resultado imposible encontrar series con resolución horaria, por lo cual se han debido calcular los períodos de retorno de 1, 2 y 3 días. Los resultados muestran que las precipitaciones máximas para 10, 30 y 50 años oscilan entre 27 y hasta $127 \mathrm{~mm}$ acumulados en 72 horas (Cuadro $N^{\circ} 3$ y Figura $\mathrm{N}^{\circ} 4$ ). Estos datos resultan ser más bajos en el caso de lluvias máximas registradas en 24 horas, si se comparan, por ejemplo, con los de la precordillera de Santiago de Chile (sobre $76 \mathrm{~mm}$ ), pero similares al realizar el mismo análisis para las 72 horas (Santiago muestra en promedio $90 \mathrm{~mm}$ ), e incluso superiores en el caso de Caquena y Chucuyo. Cabe destacar que la Figura $N^{\circ} 4$ permite advertir el aumento de la irregularidad de las precipitaciones para tres días en todo el Altiplano, pues la gráfica incrementa los altibajos entre estaciones. 
Cuadro $\mathrm{N}^{\circ} 3$

Períodos de retorno para precipitaciones máximas de 24, 48 y 72 horas obtenidos mediante la distribución Gumbel para 10, 30 y 50 años, expresados en milímetros (mm)

\begin{tabular}{|c|c|c|c|c|c|c|c|c|c|}
\hline \multirow[t]{2}{*}{ Nombre estación } & \multicolumn{3}{|c|}{$\begin{array}{c}\text { Períodos de retorno de } \\
24 \text { horas }\end{array}$} & \multicolumn{3}{|c|}{\begin{tabular}{|c|} 
Períodos de retorno de \\
48 horas \\
\end{tabular}} & \multicolumn{3}{|c|}{$\begin{array}{c}\text { Períodos de retorno de } \\
72 \text { horas } \\
\end{array}$} \\
\hline & $\mathrm{T}=10$ & $\mathrm{~T}=30$ & $\mathrm{~T}=50$ & $\mathrm{~T}=10$ & $\mathrm{~T}=30$ & $\mathrm{~T}=50$ & $\mathrm{~T}=10$ & $\mathrm{~T}=30$ & $\mathrm{~T}=50$ \\
\hline Visviri & 32 & 39 & 42 & 44 & 53 & 57 & 54 & 65 & 71 \\
\hline Caquena & 42 & 54 & 59 & 70 & 91 & 101 & 88 & 114 & 127 \\
\hline Chungará Retén & 32 & 40 & 44 & 52 & 67 & 74 & 68 & 87 & 96 \\
\hline Chungará Ajata & 30 & 35 & 37 & 43 & 50 & 54 & 50 & 56 & 59 \\
\hline Cotacotani & 30 & 36 & 39 & 47 & 57 & 62 & 61 & 75 & 82 \\
\hline Chucuyo Retén & 38 & 47 & 51 & 59 & 73 & 80 & 76 & 96 & 105 \\
\hline Parinacota (ex Endesa) & 45 & 59 & 65 & 56 & 71 & 78 & 65 & 80 & 87 \\
\hline Guallatire & 29 & 34 & 37 & 45 & 54 & 58 & 56 & 68 & 73 \\
\hline Colchane (Tte. Isluga) & 27 & 34 & 37 & 38 & 48 & 52 & 46 & 57 & 62 \\
\hline Cancosa & 28 & 35 & 38 & 43 & 53 & 58 & 51 & 65 & 71 \\
\hline Coyacagua & 27 & 33 & 36 & 39 & 49 & 53 & 46 & 57 & 62 \\
\hline
\end{tabular}

Fuente: Elaboración propia.

Figura $\mathrm{N}^{\circ} 4$

Períodos de retorno para precipitaciones máximas de 24, 48 y 72 horas obtenidos mediante la distribución Gumbel para 10, 30 y 50 años, expresados en milímetros (mm)

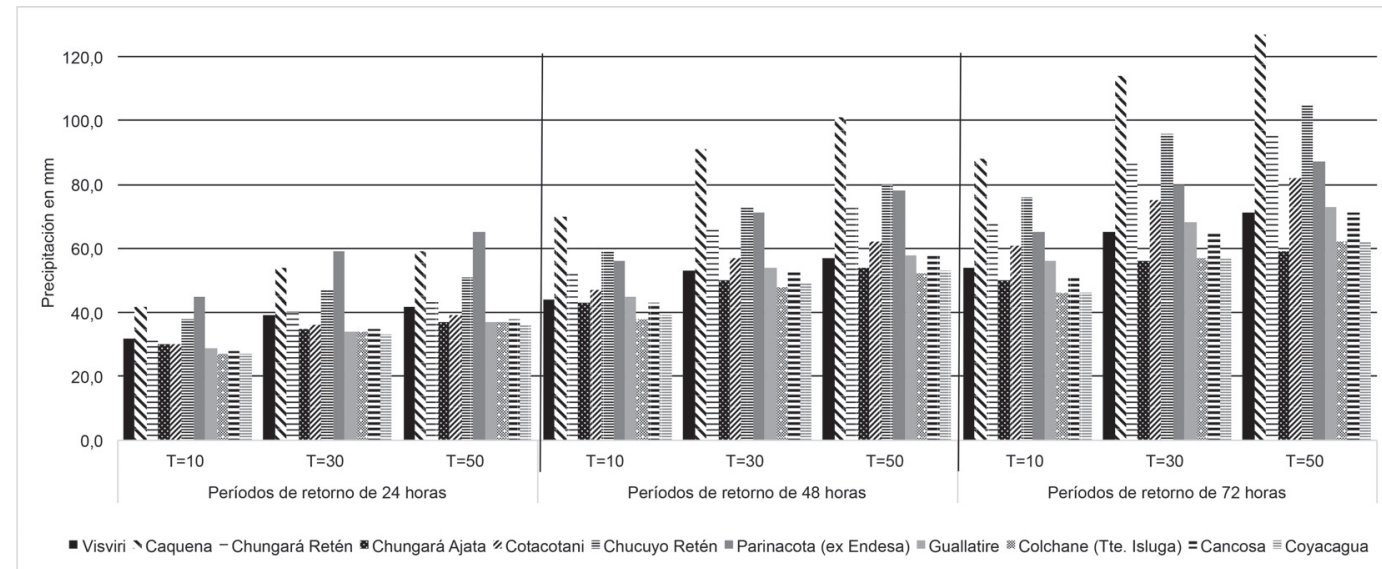

Fuente: Elaboración propia.

\section{Cambio y variabilidad climática futura}

La aplicación del método de anomalías y la evaluación de los cambios en la varia- bilidad de las precipitaciones en el período 2061-2080 permite establecer que el último IPCC (2013) prevé un aumento muy significativo de las temperaturas del Altiplano del norte de Chile (Figura $N^{\circ} 5$ ). Los cambios son muy fuertes en el extremo septentrional 
del Altiplano de Chile (cuencas de Uchusuma Caquena), para luego ser débiles entre Chungará y estero Sencata. Desde río Isluga a quebrada Carcas los cambios térmicos son fuertes, y finalmente, el extremo sur del Altiplano de la región de Tarapacá exhibe cambios débiles, es decir, menores a $2{ }^{\circ} \mathrm{C}$. De este modo, los cambios térmicos previstos para el Altiplano son mayores que aquellos esperados en zonas costeras, incluso que las sierras y precordillera altiplánica, con la excepción de lago Chungará, río Lauca, Salar de Surire y estero Sencata en la Región de Arica-Parinacota, y el Salar de Coposa y Michincha en la Región de Tarapacá.
Los cambios porcentuales de la precipitación muestran descensos fuertes y muy fuertes en el Altiplano, sobre todo en el extremo norte y sur de las regiones de Arica-Parinacota y Tarapacá. Esta "nueva realidad" que expone el AR5 (IPCC, 2013) permite diferenciar cuencas y subcuencas altiplánicas en cuanto a la disponibilidad de aguas Iluvia, que será bastante menor a la actual. Por ejemplo, en Cotacotani $(427 \mathrm{~mm})$ podría reducirse la precipitación en un $102 \%$, lo cual implica que su media en el período 2061-2080 sea de unos $210 \mathrm{~mm}$, es decir, $217 \mathrm{~mm}$ menos que las que se consiguen en promedio actualmente.

Figura $\mathrm{N}^{0} 5$

Cambios en las temperaturas hacia 2061-2080 según el escenario RCP8.5 en el Altiplano del norte de Chile

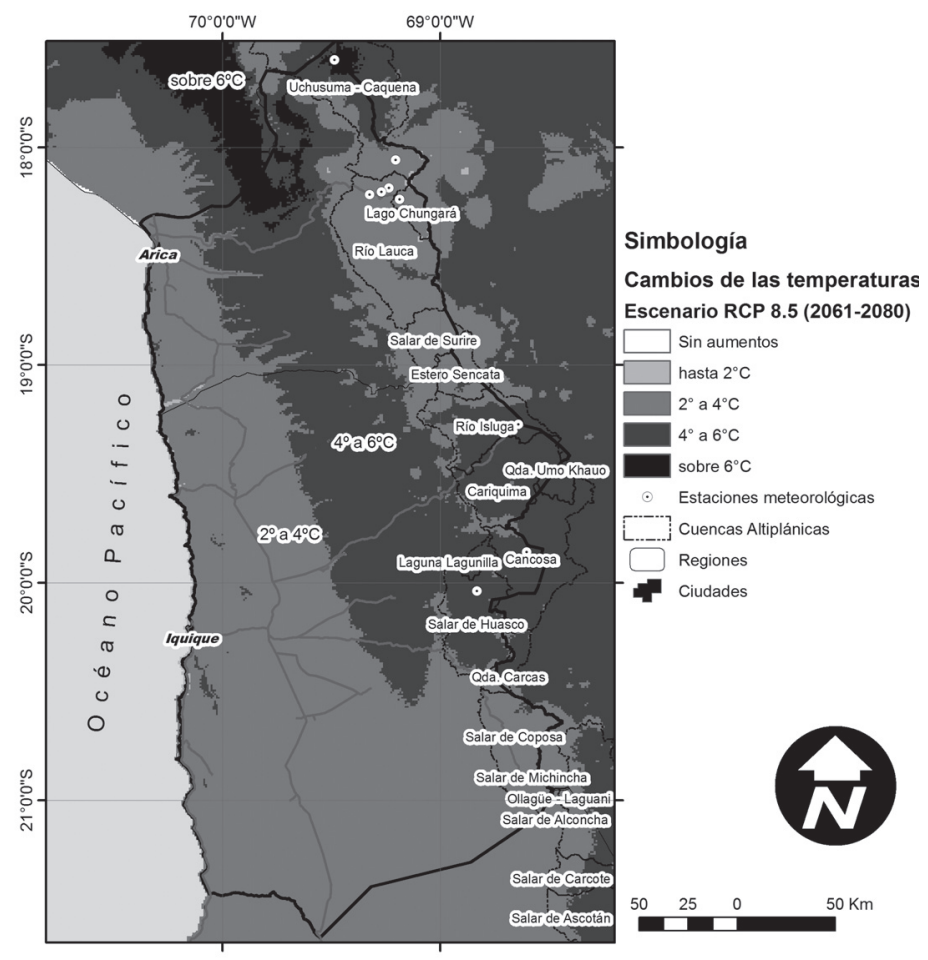

Fuente: Elaboración propia.

Un asunto poco abordado en la literatura de cambio climático hasta ahora se refiere a los posibles escenarios de variabilidad de las precipitaciones (en general solo indican aumento o disminución), es decir, información sobre cambios entre períodos extre- 
mos. Dicho esto, es posible advertir que las tendencias de las precipitaciones señalan contundentes disminuciones, pero no reportan información sobre la distribución de sus mínimos y máximos. Por ello, abordar los cambios del Coeficiente de Variación permite conocer si se deberá afrontar un clima más regular o irregular. Los resultados permiten afirmar un claro aumento del CV en el Altiplano, el cual sigue fielmente los pisos altitudinales (Figura $N^{\circ} 7$ ). Dichos cambios superan el $50 \%$, cifra considerada en climatología como muy alta. De tal modo, no será raro encontrar precipitación extrema mayor a la actual, pese a la tendencia generalizada a la disminución de su cuantía.

Al contrastar los resultados de cambio en precipitación y aplicar las regresiones entre el Coeficiente de Variación y la sequía, o incluso la pluviometría anual, se puede señalar que un aumento de un $10 \%$ en el primero puede implicar hasta 18 días más de sequía estival, y hasta $70 \mathrm{~mm}$ menos de precipitación anual. No obstante, todo indica que el CV aumentará más de $50 \%$, por lo que habrá períodos estivales sin precipitación y otros con precipitaciones máximas mayores a las extremas actuales.

Figura $\mathrm{N}^{\circ} 6$

Cambios en las precipitaciones hacia 2061-2080 según el escenario RCP8.5 en el Altiplano del norte de Chile

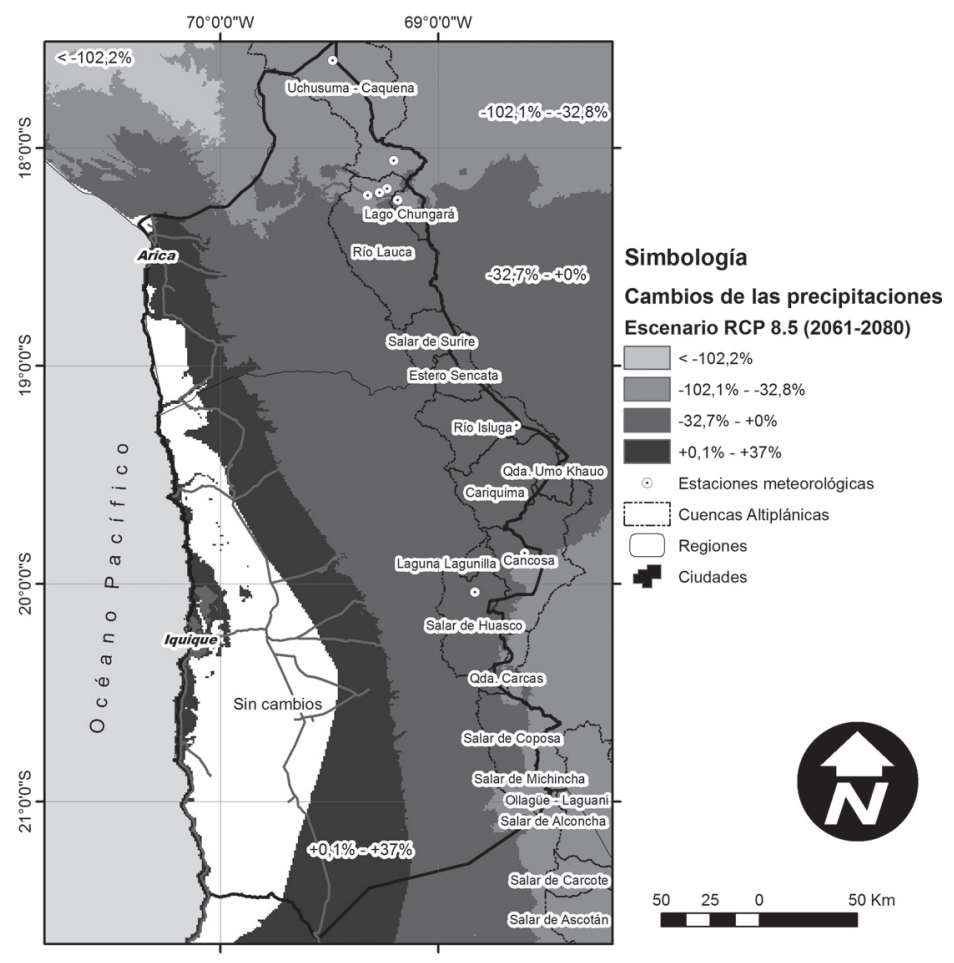

Fuente: Elaboración propia. 
Figura $\mathrm{N}^{0} 7$

Coeficiente de variación de las precipitaciones en el escenario actual (1950-2000) y según el escenario RCP8.5 (2061-2080) en el Altiplano del norte de Chile
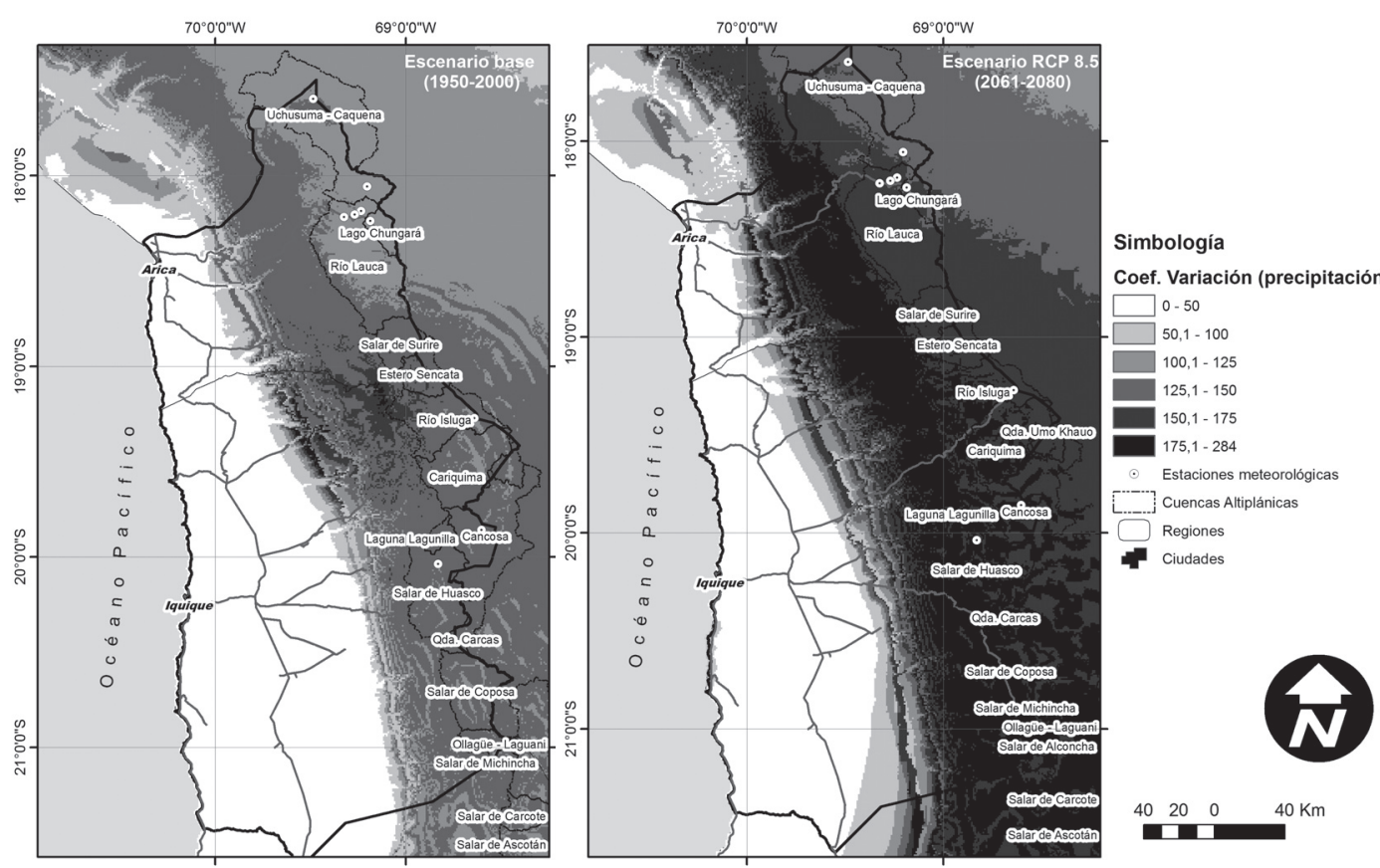

Fuente: Elaboración propia.

\section{Conclusiones}

El Altiplano del norte de Chile posee desde el punto de vista geográfico un interesante gradiente norte sur de las variables de precipitación y temperatura, lo cual implica una sección norte, que a escala de cuencas y subcuencas ofrece matices interesantes, por ejemplo en el caso de Uchusuma-Caquena, lugar donde se localiza la estación meteorológica Visviri, en comparación con las cuencas del Salar de Huasco y Lagunillas, localizadas en la sección sur (Coyacagua). Consecuentemente, no cabe esperar un comportamiento homogéneo de los climas altiplánicos, que justifique la aplicación de modelos geográficamente generalizados, lo que invalida a su vez estrategias de manejo, mitigación o adaptación que no consideren debidamente las particularidades de los ecosistemas y lugares específicos.
La alternancia de períodos húmedos y secos es observada tanto a escala diaria como interanual. A escala diaria, la longitud de los períodos secos aumenta hacia el sur (Cancosa), y los períodos lluviosos son más cuantiosos al norte (Caquena). A escala interanual, los ciclos secos pueden perdurar hasta 5 años, mientras que los de lluvia alcanzan actualmente un máximo de 3 años. Es de esperar un aumento de la longitud de ellos, lo que podría significar cambios importantes en los sistemas naturales y socioeconómicos.

El Índice de Concentración $(\mathrm{Cl})$ de precipitaciones es muy bajo $(0,50$ a 0,43$)$, incluso menor a los alcanzados en el desierto de Atacama. Esto es muy contradictorio con los otros indicadores de irregularidad, y permite plantear nuevas hipótesis, o incluso desechar a dicho indicador para esta región. No obstante, es geográficamente coherente. Es posible que una revisión a resolución horaria o 
en clases mayores a $1 \mathrm{~mm}$ permita comprender mejor la causa de la escasa representación de la irregularidad pluviométrica.

Si bien la precipitación del Altiplano del norte de Chile se concentra en verano, es posible encontrar rachas de días secos de más de un mes en dicho período (Chucuyo Retén, Colchane, Cancosa y Coyacagua), los cuales según estos resultados se pueden incrementar en cerca de 18 días en el peor escenario de cambio climático, y en el período 20612080. Ello tendría graves repercusiones en los ecosistemas de humedales y salares y obviamente, en las actividades económicas de ganadería y minería.

Los aumentos de temperatura y la disminución sostenida de la precipitación suponen un aumento de la evaporación del agua en el suelo, lo cual permite pronosticar una mayor presión sobre los recursos hídricos subterráneos, los cuales son escasamente renovables a escala humana.

La variabilidad actual de las precipitaciones torna insuficiente la posibilidad de que mediante extrapolación hacia el futuro se pueda concluir en un aumento de ella. No obstante, el último IPCC (2013) es contundente en afirmar un aumento sostenido y de gran envergadura de la variabilidad climática en el Altiplano, agravando de casi igual manera la ocurrencia de precipitación máxima extrema y de sequía, es decir, exacerbando los contrastes, a pesar de que se registren menores precipitaciones anuales.

En este sentido, el aumento de la aridez es un escenario altamente probable, por lo que se sugiere tomar medidas con tiempo de modo estratégico en una región altamente sensible al cambio climático. Esta última afirmación se ampara en cifras de calentamiento mayores a $4{ }^{\circ} \mathrm{C}$ y de una disminución de las precipitaciones por sobre un $32 \%$ respecto a las actuales.

\section{Referencias bibliográficas}

ACEITUNO, P. Aspectos generales del clima en el Altiplano sudamericano. En: Actas del II Simposio Internacional de Estudios Altiplánicos. Arica, 1993, p. 63-69.
ACEITUNO, P. Elementos del clima en el Altiplano Sudamericano. Revista GeofísicaIPGH, 1996, No 44, p. 37-55.

BI, D.; DIX, M.; MARSLAND, S.; O'FARRELL, S.; RASHID, H.; UOTILA, P.; HIRST, A.; KOWALCZYK, E.; GOLEBIEWSKI, M.; SULLIVAN, A.; YAN, H.; HANNAH, N.; FRANKLIN, C.; SUN, Z.; VOHRALIK, P.; WATTERSON, I.; ZHOU, X.; FIEDLER, R.; COLLIER, M.; MA, Y.; NOONAN, J.; STEVENS, L.; UHE, P.; ZHU, H.; GRIFFIES, S.; HILL, R.; HARRIS, C. \& PURI, K. The ACCESS coupled model: description, control climate and evaluation. Australian Meteorological and Oceanographic Journal, 2013, №63, p. 41-64.

CLARKE, R. Fitting and testing the significance of linear trends in Gumbel-distributed data. Hydrology and Earth System Sciences Discussions, 2002, №6, p. 17-24.

FALVEY, M. \& GARREAUD, R. Moisture variability over the South American Altiplano during the SALLJEX observing season. Journal of Geophysical Research, 2005, Vol. 110, No 22. Disponible en Internet: http://www.dgf. uchile.cl/rene/PUBS/altiplano_saljjex.pdf

FUENZALIDA, H.; ACEITUNO, P.; FALVEY, M.; GARREUAD, R.; ROJAS, M. \& SÁNCHEZ, P. Study on climate variability for Chile during the 21 st century. Technical Report prepared for the National Environmental Committee. Santiago de Chile, 2007.

FUENZALIDA, H. y RUTLANT, J. Origen del vapor de agua que precipita en el Altiplano de Chile. Anales del II Congreso Interamericano de Meteorología y $V$ Congreso Argentino de Meteorología. Buenos Aires, 1987, p. 631-634.

GARREAUD, R. \& ACEITUNO, P. Interannual rainfall variability over the South American Altiplano. Journal of Climate, 2001, $N^{\circ} 14$, p. 2779-2789.

GARREUD, R.; VUILLE, M. \& CLEMENT, A. The climate of the Altiplano: Observed current conditions and past change mechanisms. Palaeogeography, Palaeoclimatology, Palaeoecology, 2003, No 194, p. 5-22. 
HIJMANS, R.; CAMERON, S.; PARRA, J.; JONES, P. \& JARVIS, A. Very high resolution interpolated climate surfaces for global land areas. International Journal of Climatology, 2005, No25, p. 1965-1978.

INTERGOVERNMENTAL PANEL ON CLIMATE CHANGE (IPCC). Climate Change 2013: The Physical Science Basis. In: STOCKER, T.F.; QIN, D.; PLATTNER, G.-K.; TIGNOR, M.; ALLEN, S.K.; BOSCHUNG, J.; NAUELS, A.; XIA, Y.; BEX, V. \& MIDGLEY, P.M. (editors). Contribution of Working Group I to the Fifth Assessment Report of the Intergovernmental Panel on Climate Change. Cambridge/New York: Cambridge University Press, 2013, p. 1535.

KARL, T.; MEEHL, G.; PETERSON, T.; KUNKEL, K.; GUTOWSKI JR, W.; EASTERLING, D. Executive Summary in Weather and Climate Extremes in a Changing Climate. Regions of Focus: North America, Hawaii, Caribbean, and U.S. Pacific Islands. In: KARL, T.R.; MEEHL, G.A.; MILLER, C.D.; HASSOL, S.J.; WAPLE, A.M. \& MURRAY, W.L. (editors). A Report by the U.S. Climate Change Science Program and the Subcommittee on Global Change Research. Washington, 2008.

MARTÍN-VIDE, J. Análisis de la irregularidad de la precipitación diaria en el litoral mediterráneo de la Península Ibérica. Revista de Geofísica, 1984, № 40, p. 101-106.

MARTÍN-VIDE, J. \& GÓMEZ, L. Regionalization of peninsular Spain based on the length of dry spells. International Journal of Climatology, 1999, N 19, p. 537-555.

MARTÍN-VIDE, J.; PROHOM, M.; BOHIGAS, M.; PEÑA, J.; ESTEBAN, P. y MONTSERRAT, D. Índices de irregularidad temporal y dimensión fractal de la precipitación anual en España. En: PÉREZ-CUEVA, E.; LÓPEZ BAEZA, A. y TAMAYO, J. (editores). El Tiempo del Clima. Buñol: AEC i Garmas Impresores, 2001, p. 157-166.

MARTÍN-VIDE, J. El tiempo y el clima. Barcelona: Rubes Editorial, 2003, p. 127.
MARTÍN-VIDE, J. Spatial distribution of daily precipitation concentration index in Peninsular Spain. International Journal of Climatology 2004, N²4, p. 959-971.

MINVIELLE, M. \& GARREUD, R. Projecting rainfall changes over the South American Altiplano. Journal of Climate, 2011, $\mathrm{N}^{\circ} 24$, p. 4577-4583.

PLISCOFF, P.; LUEBERT, F.; HILGER, $\mathrm{H}$. \& GUISAN, A. Effects of alternative sets of climatic predictors on species distribution models and associated estimates of extinction risk: A test with plants in an arid environment. Ecological Modelling, 2014, № 288, p. 166-177

ROMERO, H.; SMITH, P.; MENDONÇA, M. y MÉNDEZ, M. Macro y mesoclimas del altiplano andino y desierto de Atacama: desafíos y estrategias de adaptación social ante su variabilidad. Revista de Geografía Norte Grande, 2013, N 55, p. 19-41.

SARRICOLEA, P.; HERRERA, M. y ARAYA, C. Análisis de la concentración diaria de las precipitaciones en Chile central y su relación con la componente zonal (subtropicalidad) y meridiana (orográfica). Investigaciones Geográficas, 2013, №45, p. 37-50.

SARRICOLEA, P. y MARTÍN-VIDE, J. Distribución espacial de las precipitaciones diarias en Chile mediante el índice de concentración a resolución de $1 \mathrm{~mm}$, entre 1965-2005. En: RODRÍGUEZ, C.; CEBALLOS, A.; GONZÁLEZ, N.; MORÁN., E. y HERNÁNDEZ, A. Cambio climático, extremos e impactos. Salamanca: Publicaciones de la Asociación Española de Climatología, 2012, p. 631-639

WILBY, R. \& WIGLEY, T. Downscaling general circulation models output: a review of. Progress in Physical Geography, 1997, Vol. 4, $\mathrm{N}^{\circ} 21$, p. 530-548. 
